\title{
ILCEA
}

Revue de l'Institut des langues et cultures

d'Europe, Amérique, Afrique, Asie et Australie

$27 \mid 2016$

Approches ergonomiques des pratiques

professionnelles et des formations des traducteurs

\section{Traducteurs à l'œuvre : une perspective ergonomique en traductologie appliquée}

Translators at Work: an Ergonomic Perspective in Applied Translation Studies

Élisabeth Lavault-Olléon

\section{(2) OpenEdition}

\section{Journals}

Édition électronique

URL : http://journals.openedition.org/ilcea/4051

DOI : 10.4000/ilcea.4051

ISSN : 2101-0609

Éditeur

UGA Éditions/Université Grenoble Alpes

Édition imprimée

ISBN : 978-2-84310-336-0

ISSN : 1639-6073

Référence électronique

Élisabeth Lavault-Olléon, « Traducteurs à l'œuvre : une perspective ergonomique en traductologie appliquée », ILCEA [En ligne], 27 | 2016, mis en ligne le 08 novembre 2016, consulté le 01 mai 2019. URL : http://journals.openedition.org/ilcea/4051 ; DOI : 10.4000/ilcea.4051

Ce document a été généré automatiquement le 1 mai 2019

(C) ILCEA 


\title{
Traducteurs à l'œuvre : une perspective ergonomique en traductologie appliquée
}

Translators at Work: an Ergonomic Perspective in Applied Translation Studies

\author{
Élisabeth Lavault-Olléon
}

1 Discipline universitaire née dans les années 1970, la traductologie prend son autonomie dans les années 1980, devenant un champ de recherche en soi, non comme réflexion sur la langue ou la littérature à partir d'exemples de traduction, mais comme « réflexion de la traduction sur elle-même à partir de sa nature d'expérience » (Berman, 1985 : 39 ).

Depuis l'article fondateur de Holmes (1988, $1^{\text {re }}$ version 1972) établissant le nom, la nature et une première cartographie des Translation Studies, le tableau représentant les différentes branches des études traductologiques s'est considérablement complexifié, comme le montre l'ontologie réalisée par Vandepitte (2008). Après des recherches qui faisaient la part belle à la traduction comme produit, l'objet d'étude s'est déplacé de la langue au discours, du produit au processus, tout en prenant graduellement en compte non seulement les relations de causalité liées à la fonction et à l'effet des traductions mais aussi la situation de communication professionnelle et ses acteurs.

L'essor des besoins en traduction dus à l'internationalisation croissante des échanges a conduit dans les années 1990 à la multiplication des formations de traducteurs ${ }^{1}$ dans des centres universitaires qui ont valorisé la traduction comme objet de recherche. L'orientation de plus en plus compétitive des universités et les pressions quantitatives sur les publications ont conduit à une explosion des recherches en traductologie. Illustrations évidentes : la publication d'une première Encyclopedia of Translations Studies (Baker, 1998) présentant un panorama des recherches en traductologie, puis celle plus récente du Handbook of Translation Studies (Gambier, 2010).

4 Une sorte de palier semble toutefois atteint dans les années 2000 en ce qui concerne les recherches théoriques pures, prolifiques dans les années 1980 et 1990, ainsi que les recherches descriptives (Toury, 1995) qui ont abondamment décrit la traduction comme 
produit, comme processus et comme fonction. Avec la révolution technologique, les perspectives ont changé : même si le poids de la traduction littéraire reste élevé tant dans la culture savante que dans la culture populaire, la demande de traduction de textes pragmatiques (Delisle, 1993:22; Froeliger, 2013:31) est devenue si prépondérante en termes quantitatifs que les formations de traducteurs se sont adaptées pour y répondre, incitant naturellement les enseignants-chercheurs à se pencher sur les nouvelles pratiques professionnelles et à chercher de nouveaux éclairages pour les analyser.

\section{Nouvelles perspectives en traductologie appliquée}

5 Les pratiques de la traduction pragmatique (spécialisée, le plus souvent) ont en effet considérablement évolué depuis les années 2000, en réponse à de nouvelles réalités. Ainsi, la mondialisation a créé une demande exponentielle en traduction à laquelle les ressources humaines disponibles sont incapables de répondre. Le développement des outils d'aide à la traduction (au sens large, englobant plus que les mémoires de traduction) et celui de la traduction automatique statistique visent à combler ce manque et ont permis d'accroître la productivité des traducteurs, y compris par l'automatisation d'une partie du travail. Parallèlement, Internet et les réseaux sociaux ont transformé l'activité de traduction et les relations interpersonnelles au travail.

6 Pour prendre en compte ces évolutions, les chercheurs se tournent vers de nouvelles orientations, qui sont à la fois descriptives et appliquées car ces deux branches distinguées par Holmes, puis par Toury, sont de moins en moins séparées. Il est en effet difficile de décrire les modifications de l'activité des traducteurs et du processus de traduction lui-même, en lien avec les ressources et outils utilisés aujourd'hui, sans être tenté d'intervenir pragmatiquement sur la formation des traducteurs en se fondant sur ces recherches descriptives.

7 La branche dite « appliquée » du descriptif de Holmes était décrite assez rapidement dans son article car ses quatre sous-branches étaient à l'époque peu développées. De façon visionnaire, Holmes en a souligné l'extrême importance pour l'avenir. Il a notamment constaté que, dans la première sous-branche "teaching", c'est la formation des traducteurs, et non l'utilisation de la traduction dans l'enseignement des langues étrangères, qui devrait requérir le plus d'attention. Il a également noté l'importance des outils, même s'il n'avait pas conscience de tous les futurs développements de l'informatique. Il a mentionné la nécessité de réfléchir à une évaluation objective des traductions dans la sous-branche " translation criticism »; et enfin, dans la sous-branche " translation policy", il a isolé " the place and role of translators, translating, and translations in society at large » (Holmes, $1988: 182$ ).

Or ce sont justement ces aspects appliqués qui méritent de plus en plus l'attention des chercheurs: le renouveau de la pédagogie dans le cadre de la formation professionnelle des traducteurs et des recherches sur les compétences ; la prise en compte des outils issus du traitement automatique des langues (TAL) et leur impact sur l'activité du traducteur humain devenu «biotraducteur "; le besoin de fonder objectivement l'évaluation, aussi bien dans la formation, le recrutement que dans les tests de systèmes automatiques; et enfin la nécessité de redéfinir non seulement l'impact de la traduction comme produit et comme activité, mais aussi la place, le rôle et le statut des traducteurs. 
9 Le tableau de Holmes a été commenté par de nombreux chercheurs, notamment par Chesterman qui regrette l'absence d'une branche d'études qu'il appelle "Translator Studies» qui serait axée non pas sur le produit, le processus ou la fonction mais sur le traducteur lui-même. Transverse à plusieurs sous-branches des trois grandes catégories présentées par Holmes, cette branche de la traductologie serait alors divisée entre aspects culturels (éthique, idéologie, tradition, histoire des traducteurs et interprètes), cognitifs (processus mentaux, normes et émotions) et sociologiques (comportements, réseaux, statuts, organisation du travail, relations aux autres et aux outils) (Chesterman, $2009: 19$ ).

Comme souvent, une fois qu'une discipline autonome a établi ses fondamentaux de façon solide, son renouvellement passe par l'éclairage d'autres disciplines. On peut voir dans la traductologie une polydiscipline (Gambier, $2006: 29)$ qui s'est construite en empruntant à d'autres disciplines telles que la linguistique, l'herméneutique, la pragmatique, ou les études culturelles, entre autres, et dont l'une des orientations récentes serait la sociologie. Axée autant sur le traducteur (profil et carrière) que sur les aspects sociétaux des métiers et des produits de l'activité (Gambier, 2006 : 39), celle-ci va jusqu'à définir une véritable sociologie de la traduction (Wolf \& Fukari, 2007).

11 Si l'orientation sociologique semble pertinente, son apport parait cependant insuffisant pour englober l'ensemble des facteurs environnementaux qui ont pris une place importante dans l'activité de traduction du fait de l'utilisation massive des technologies et des nouvelles organisations du travail. Même les recherches cognitivistes sur la traduction soulignent que la traduction est une activité « située » et que le rôle joué par l'environnement social, physique et matériel du traducteur doit être pris en compte au même titre que ce qui se passe dans son cerveau (Risku, $2002: 530$ ).

12 Nous pensons que la traductologie est une discipline spécifiquement humaine qui ne se réduira jamais à l'analyse de systèmes linguistiques visant à l'automatisation complète des transferts linguistiques. Sa "nature d'expérience » reste inscrite dans ses gènes. Il nous semble aussi que les recherches descriptives devraient être conduites dans un but pragmatique, rejoignant alors les recherches appliquées. Pour rester centrées sur le traducteur humain, dans la ligne des «Translator Studies» de Chesterman, celles-ci peuvent bénéficier de l'apport d'une discipline ayant justement pris l'humain au travail comme objet d'études, l'ergonomie.

\section{L'approche ergonomique}

13 Aujourd'hui, la traduction peut être vue comme un système complexe de production de contenus multilingues, englobant non seulement des acteurs et des compétences multiples mais aussi des interactions homme-machine et des facteurs environnementaux. Les traducteurs doivent remplir des tâches diverses qui exigent des compétences linguistiques de compréhension et de production, des compétences avancées en extraction et gestion de l'information et un haut niveau de compétence dite instrumentale pour à la fois interagir avec les clients, manipuler des outils, sélectionner les sources d'information et gérer les contraintes organisationnelles. Le poste de travail est informatisé, conçu pour augmenter la productivité, améliorer la cohérence et réduire les délais et les coûts. Mais l'environnement matériel n'est souvent pas approprié à du travail intensif sur écran et le système organisationnel mal adapté. De nouvelles tâches sont apparues, si fortement qu'elles deviennent de nouveaux métiers (la post-édition, par 
exemple). Avec l'utilisation maximale des outils, elles donnent lieu à des interrogations cruciales sur l'interaction homme-machine mais aussi sur le rôle, le statut et l'avenir de la profession de traducteur.

Il est donc nécessaire d'analyser tous ces facteurs qui ont une influence sur le travail, et incidemment, sur le bien-être et l'identité des traducteurs. Définie lors du IV ${ }^{e}$ Congrès international d'ergonomie (1969) comme «l'étude scientifique de la relation entre l'homme et ses moyens, méthodes et milieux de travail », l'ergonomie nous apporte un cadre pertinent, permettant d'analyser les aspects cognitifs, physiques et organisationnels des pratiques professionnelles, y compris dans la formation.

En 2010, à l'université de Grenoble, nous avons organisé un premier colloque, qui a ouvert la voie à l'exploration des nombreuses interfaces entre la traduction et l'ergonomie et a suscité des publications sur le sujet (entre autres, Brunette \& O'Brien, 2011 ; Ehrenberger et O'Brien, 2015 ; Lavault-Olléon, 2011 ; Massey \& Ehrensberger-Dow, 2011). Le deuxième colloque de Grenoble, organisé en mars 2015, a contribué à nourrir cette thématique, et depuis, l'ergonomie est devenue une thématique de grands colloques internationaux en traductologie : elle figure notamment dans le programme du congrès de l'EST, European Society for Translation Studies (Aarhus, 15-16 septembre 2016) et dans celui du Congrès mondial de la traductologie (Paris, 10-14 avril 2017).

Les articles de ce numéro 27 de la revue ILCEA reflètent en partie les travaux abordés lors du colloque de mars 2015 à Grenoble, "Traducteurs à l'œuvre», dont la thématique principale était l'analyse ergonomique des pratiques professionnelles en traduction, ainsi que ses implications sur la formation. Dans une optique de formateur, à la fois descriptive et appliquée, qui est celle de nombreux universitaires, il est utile d'analyser comment les nouvelles conditions de travail et les modifications de l'environnement professionnel influent sur les traducteurs et sur leurs performances, et comment ces changements peuvent être abordés aux différents niveaux de la formation initiale et continue. Les huit articles qui suivent abordent cette question sous différents angles : trois articles portent sur l'ergonomie physique, c'est-à-dire l'environnement matériel du travail, ses effets sur la santé et la remédiation possible; trois abordent l'ergonomie cognitive et organisationnelle, que ce soit par l'effet des tâches de post-édition sur la qualité des traductions ou la nécessité d'organiser des pratiques collaboratives entre traducteurs spécialisés et experts ou encore les relations entre sociologie et traductologie dans les recherches récentes; et pour finir, deux articles traitent de l'adéquation entre pratiques professionnelles et formation.

\section{Traducteurs à l'œuvre : éclairages ergonomiques}

Dans ce numéro de la revue ILCEA consacré aux approches ergonomiques des pratiques professionnelles et des formations des traducteurs, c'est l'ergonomie physique qui ouvre la thématique. Encore balbutiantes en 2010, les recherches sur l'ergonomie physique appliquée à la traduction ont donné lieu à d'importantes investigations sur les conditions de travail des traducteurs et leurs effets, menées notamment par les chercheurs de l'Institut de traduction et d'interprétation à l'université des sciences appliquées de Zurich (ZHAW), qui se sont associés à ceux de l'Institut d'ergonomie de la même université. Ces enquêtes ont été réalisées à la fois au niveau international et au plus près des traducteurs d'une région. 

en ligne anonyme dans le cadre d'un projet interdisciplinaire qui avait pour but de recueillir des informations sur la façon dont les traducteurs à travers l'Europe ont agencé et organisé leur poste de travail. Accessible en six langues (français, anglais, allemand, italien, portugais et espagnol), le questionnaire relatif à cette étude a été envoyé par des organisations professionnelles qui ont relayé la demande d'informations dans les réseaux. L'intérêt suscité par la question au sein de la communauté professionnelle se reflète dans le grand nombre de questionnaires complétés (1 850), mais aussi dans les commentaires formulés par les répondants dans l'étude elle-même et dans les courriels adressés à l'équipe de chercheurs. Le premier article, écrit par Maureen Ehrensberger-Dow, Andrea Hunziker Heeb et Gary Massey, associés à Ursula Meidert, Silke Neumann et Heidrun Becker, expose les résultats de l'étude et met l'accent sur les ressemblances et disparités des pratiques selon que les traducteurs sont salariés en entreprise, employés par des organisations publiques ou travaillant comme indépendants, dans les différents pays. L'enquête extrêmement minutieuse dresse un tableau complet du poste de travail (mobilier, espace disponible, ambiance sonore et lumineuse, possibilités d'isolement du bruit) mais aussi du degré de pénétration des technologies d'aide à la traduction (écrans, périphériques, outils de TAO), de l'organisation du travail (processus individuel, workflow, autonomie) et des problèmes de santé liés aux pratiques professionnelles. Les résultats montrent qu'il existe une marge d'amélioration non négligeable dans le poste de travail et l'organisation, notamment chez les traducteurs indépendants, nettement moins bien équipés que les autres. L'enquête a participé à la sensibilisation des traducteurs aux aspects ergonomiques de leur activité et conclut sur la nécessité d'inclure ces questions dans la formation. traducteurs professionnels occidentaux restent assis de longues heures devant leur écran. Une deuxième enquête, effectuée principalement par les ergothérapeutes Ursula Meidert, Silke Neumann et Heidrun Becker, associées à la traductologue Maureen EhrensbergerDow, a consisté en des évaluations et interviews réalisées en Suisse et axées sur l'ergonomie du poste de travail et sur les problèmes de santé associés. Les chercheuses ont observé les postes de travail de 36 traducteurs et traductrices professionnels installés à leur compte ou engagés dans des institutions ou des entreprises commerciales. Chaque poste de travail a fait l'objet d'une évaluation et d'une comparaison avec les recommandations en matière d'ergonomie fournies par la littérature spécialisée. Des interviews ont, en outre, été menées avec les traducteurs à l'issue des évaluations dans le but de connaitre leur état de santé et d'éventuels problèmes attribués à leur activité professionnelle. Des résultats extrêmement détaillés sont présentés sur chaque problème de santé, croisés en fonction du sexe, du statut et de l'équipement du poste de travail. Une grande partie des postes évalués présentent un équipement et un mobilier dotés d'un niveau élevé d'ergonomie, mais l'agencement n'est pas toujours adapté aux besoins de la personne assignée au poste de travail. Même si, dans l'ensemble, le nombre de problèmes de santé exprimés reste faible, la majorité d'entre eux concernent la fatigue oculaire ainsi que les troubles musculo-squelettiques du dos, des épaules, des bras et des mains. L'article propose un certain nombre d'améliorations possibles du poste de travail, y compris le recours à un ergonome professionnel dans la mesure du possible. L'objectif est aussi de sensibiliser l'auditoire à l'agencement adéquat du poste de travail dans le cadre de formations professionnelles. 
20 Après ces deux articles exposant des résultats d'enquêtes très détaillées sur l'activité des traducteurs, la parole est donnée à une traductrice, Lucia Peters-Geiben, qui occupe le poste de correspondant ergonomie au sein de la Direction générale de la traduction (DGT) à la Commission européenne. Cette auteure expose de façon très concrète comment les habitudes de travail d'un département de traduction d'une grande institution peuvent être modifiées à force de persévérance et de motivation, avec l'appui de ressources non négligeables. Elle explique quelques principes ergonomiques de base qu'il est important de connaître et de mettre en pratique : ses recommandations portent sur la prévention dite " contextuelle», entre autres le bon choix du mobilier de bureau et de l'équipement informatique, mais aussi sur la prévention comportementale, qui se traduit par l'appropriation de ces principes, l'utilisation correcte de cet équipement et la mise en place de pratiques permettant de préserver le bien-être tout au long de la journée de travail et au-delà. L'article porte également sur la façon d'appliquer une véritable politique ergonomique dans une institution employant des traducteurs. L'auteure recommande l'intégration des problématiques de l'ergonomie dans la formation des traducteurs et dans l'ensemble de l'université de façon plus globale, du point de vue théorique et pratique, et dans une perspective de long terme.

21 Après ces trois articles traitant principalement d'ergonomie physique, mais comprenant aussi des aspects cognitifs et organisationnels, c'est plus précisément l'impact des outils sur le processus cognitif et sur la qualité du travail qui est abordé dans le quatrième article. Hanna Martikainen et Natalie Kübler, de l'université Paris Diderot, y présentent une étude de cas sur l'activité de post-édition dans une entreprise privée. Cette étude montre comment la nature même du processus associant traduction automatique et postédition humaine influence profondément les caractéristiques des textes ainsi produits, en raison de l'ergonomie cognitive propre à cette interaction homme-machine. Par exemple, l'automatisation d'une partie du processus traductionnel perturbe, via l'interposition de la sortie de traduction automatique, l'application des stratégies de traduction habituelles par le post-éditeur. Les consignes de post-édition minimalistes, privilégiant la rapidité aux dépens des considérations stylistiques, tout comme l'accoutumance à la sortie de traduction automatique, renforcent encore cette interférence avec la langue source. Les améliorations que peut apporter l'automatisation en termes de cohérence terminologique ou de simplification phraséologique sont limitées par les erreurs de langue ou d'interprétation induites, entre autres, par le caractère fragmentaire de l'activité de postédition. Les auteures explorent comment les textes issus de ce nouveau mode de production diffèrent de ceux produits par la traduction humaine ou biotraduction, et en déduisent la nécessité de former les post-éditeurs aux spécificités de cette tâche et aux risques d'erreur qu'elle comporte.

Les outils d'aide à traduction sont non seulement les mémoires de traduction ou les logiciels de traduction automatique, mais aussi les ressources numérisées nécessaires aux traducteurs spécialisés, et notamment les outils terminologiques. Michel Rochard et Caroline Champsaur prennent l'exemple de leur service de traduction à l'OCDE (Organisation de coopération et de développement économiques), dans lequel les traducteurs et les experts des domaines économique et financier collaborent pour optimiser les ressources terminologiques. Les outils collaboratifs sont l'un des aspects étudiés par l'ergonomie organisationnelle mais leur impact cognitif est également remarquable, et c'est ce que montre cet article : l'auteur-expert et le traducteur spécialisé ont un rapport différent au monde et aux mots, le premier choisissant des mots pour 
décrire une réalité complexe alors que le second a besoin de comprendre quel concept ou découpage du monde correspond aux mots de l'expert. Les deux démarches sont complémentaires, l'expert et le traducteur étant tous deux des «travailleurs de la connaissance ». Pour accroître l'impact de ses analyses et de ses réflexions, l'expert doit non seulement les partager, mais aussi fournir les clefs nécessaires à la compréhension de son travail, et s'assurer de la transmission de son expertise, à la fois par la traduction et par un stockage intelligent de ses connaissances, que permet le travail terminologique du traducteur.

Le développement d'une culture numérique du partage (multiplication des réseaux sociaux comme outils de communication de masse) permet de nouvelles formes de collaboration, comme le portail terminologique collaboratif AGORA que nous présentent Michel Rochard et Caroline Champsaur: une plateforme qui répond aux besoins de l'expert (faire connaître son travail et en améliorer l'impact) et à ceux du traducteur (utiliser et conserver des définitions fiables). En rassemblant les données spécialisées et en plaçant le concept au centre de l'entrée terminologique, le portail collaboratif est un exemple d'ergonomie cognitive partagée. Pour les traducteurs, c'est un formidable vecteur de formation spécialisée et notamment de formation continue.

L'ergonomie organisationnelle traite des organisations du travail au sein d'une structure et de son impact sur la santé et le bien-être des acteurs. C'est la branche de l'ergonomie qui se rapproche le plus du tournant sociologique que nous avons mentionné plus haut. Dans l'article suivant, Anna Kuznik, enseignante-chercheuse à l'université de Wroclaw, explore justement ces rapports entre trois disciplines scientifiques connexes, à savoir l'ergonomie (organisationnelle notamment), la sociologie du travail et la traductologie. Ces trois disciplines partagent le même objet d'étude (le travail), les méthodes de recherche mises en œuvre (l'observation et l'enquête) et les difficultés particulières liées aux exigences de la recherche menée sur le terrain, par exemple au sein d'organisations à but lucratif telles que les entreprises. Anna Kuznik présente un état de l'art de cette question, en effectuant une revue bibliographique des principales études effectuées dans chaque domaine et en montrant leurs apports méthodologiques. Elle y intègre la présentation d'un projet de recherche interdisciplinaire fondé sur l'observation des pratiques de traducteurs dans des entreprises prestataires de services en traduction établies dans trois pays européens. Son objectif est de décrire l'organisation des tâches et de mieux cerner le sens et la place de l'activité de traduction proprement dite dans ces organisations.

L'article présenté par Cécile Frérot et Lionel Karagouch de l'Université Grenoble Alpes porte spécifiquement sur la formation des traducteurs en rapport avec les outils utilisés par les professionnels. Comme il a déjà été souligné, l'environnement de travail du traducteur, de plus en plus intégré, entraîne des modifications considérables de l'activité de traduction, auxquelles les étudiants doivent être préparés. Il est donc nécessaire d'incorporer l'environnement quotidien du traducteur dans les formations universitaires afin de proposer des contenus le plus possible en adéquation avec la réalité professionnelle. L'article propose une caractérisation des outils d'aide à la traduction, afin de clarifier la terminologie de la TAO, tout en soulignant les caractéristiques ergonomiques de ces outils. Plusieurs enquêtes avaient déjà mis en évidence les pratiques professionnelles en matière d'outils d'aide à la traduction. Les auteurs ont mené deux enquêtes spécifiques auprès d'universités formant des traducteurs et auprès de jeunes diplômés de master. Ils en déduisent que, pour les formations enquêtées, les pratiques 
universitaires et professionnelles sont plutôt en adéquation et bien ancrées dans le quotidien des (futurs) traducteurs. La question de l'exploitation de corpus est également posée, ceux-ci, très prisés par les universitaires, étant mal connus et peu utilisés par les professionnels pour diverses raisons, notamment économiques.

L'utilisation des corpus électroniques dans la formation des traducteurs est principalement le sujet de l'article de Rudy Loock, de l'université de Lille, qui note lui aussi la disparité entre les travaux universitaires préconisant l'exploitation de corpus comme aide à la traduction et le fait que le recours aux corpus électroniques chez les traducteurs demeure rare. Les corpus n'ont en effet pas acquis le statut d'outil d'aide à la traduction alors qu'ils sont omniprésents dans les outils utilisés par les traducteurs (mémoires de traduction, outils de traduction automatique, sites internet de concordances très utilisés), même si le terme " corpus » n'y apparaît pas. Parallèlement, d'autres types de corpus ne sont ni connus ni utilisés, comme les mini-corpus spécialisés en langue cible ou encore les corpus comparables alignant des textes non-traduits de même nature rédigés dans deux langues. L'une des raisons de cette non-utilisation des corpus comme outil d'aide à la prise de décision en traduction est leur manque d'ergonomie : leur exploitation est souvent fastidieuse du fait de contenus et d'interfaces très hétérogènes tandis que les outils commerciaux semblent bien plus simples d'utilisation. L'auteur montre que la compilation et l'exploitation de corpus spécifiques peut être un atout pour les traducteurs, d'abord comme aide à la compréhension mais aussi et surtout pour les aider à trouver une expression naturelle en langue cible, en s'appuyant sur les collocations et les structures syntaxiques spontanées de la langue cible. Notant que les linguistes et les traducteurs n'ont pas les mêmes attentes des corpus, il plaide pour une adaptation ergonomique des corpus qui permettrait leur exploitation rapide par les traducteurs.

L'approche ergonomique est le fil conducteur de ces articles qui éclairent les pratiques professionnelles actuelles. Certains montrent que les conditions de travail, qu'elles soient matérielles, cognitives ou organisationnelles, peuvent conduire à des situations préoccupantes, ayant des répercussions directes sur la santé des personnes et sur la qualité des traductions. D'autres proposent des améliorations et présentent des situations où le traducteur peut prendre en main son environnement, par la prévention et la sensibilisation ou par la collaboration active entre différents acteurs. Les formateurs de traducteurs sont en tout cas incités à prendre en compte l'environnement réel du « traducteur à l'œuvre » et à sensibiliser dès l'apprentissage les futurs professionnels à l'importance de l'ergonomie.

\section{BIBLIOGRAPHIE}

BAKER Mona (1998), Routledge Encyclopaedia of Translation Studies, Londres/New York : Routledge.

BERMAN Antoine (1989), « La traduction et ses discours », Meta : journal des traducteurs/Meta:

Translators' Journal, 34(4), 672-679, <http://id.erudit.org/iderudit/002062ar>. 
BRUNETTE Louise \& O'BRIEN Sharon (2011), « Quelle ergonomie pour la pratique postéditrice des textes traduits? », ILCEA (14), <http://ilcea.revues.org/1081>.

Chesterman Andrew (2009), « The Name and Nature of Translator Studies », Hermes (42), 13-22, <http://download1.hermes.asb.dk/archive/download/Hermes-42-2-chesterman_net.pdf>. DeLISLE Jean (1993), La Traduction raisonnée, Ottawa : Les Presses de l'Université d'Ottawa.

EHRENSBERGER-DOW Maureen \& O'BRIEN Sharon (2015), « Ergonomics of the translation workplace: Potential for cognitive friction », Special Issue of Translation Spaces, 4(1), 98-118.

FROELIGER Nicolas (2013), Les Noces de l'analogique et du numérique - De la traduction pragmatique, Paris : Les Belles Lettres.

GAMBIER Yves (2006) « Pour une socio-traduction », J. Ferreira Duarte, A. Assis Rosa \& T. Seruya (dir.), Translation Studies at the Interface of Disciplines, Amsterdam/ Philadelphie : John Benjamins Publishing Company.

GAMBIER Yves \& van DoorsLAER Luc (2010-2015), Handbook of Translation Studies (vol. 1-6), Amsterdam/Philadelphie : John Benjamins Publishing Company, <https://benjamins.com/ \#catalog/online\%20resource/ehts/main>.

HoLmes James S. (1988), « The Name and Nature of Translation Studies », J. S. Holmes, Translated! Papers on Literary Translation and Translation Studies, Amsterdam : Rodopi, 67-80 (article republié dans L. Venuti (2000), The Translation Studies Reader, Londres : Routledge, 172-185).

LAVAULT-OLLÉON Élisabeth (2011), « L'ergonomie, nouveau paradigme pour la traductologie », ILCEA (14), <http://ilcea.revues.org/1078>.

MASSEY Gary \& EHRENBERGER-Dow Maureen (2011), « Technical and Instrumental Competence in Translation: Ergonomic Aspects of Workplace Processes and Practices », ILCEA (14), <http:// ilcea.revues.org/1060>.

RISKU Hanna (2002), « Situatedness in Translation Studies », Cognitive Systems Research, 3(3), 523-533.

TOURY Gideon (1995), Descriptive Translation Studies and Beyond, Amsterdam/Philadelphie : John Benjamins Publishing Company.

VANDEPITTE Sonia (2008), « Remapping Translation Studies: Towards a Translation Studies Ontology », Meta : journal des traducteurs/Meta: Translators' Journal, 53(3), 569-588, <http:// www.erudit.org/revue/meta/2008/v53/n3/019240ar>.

WOLF Michaela \& FUKARI Alexandra (2007), Constructing a Sociology of Translation, Amsterdam/ Philadelphie : John Benjamins Publishing Company.

\section{NOTES}

1. Dans le présent article, conformément aux normes du français, le masculin singulier «traducteur» représente le métier, même si celui-ci est majoritairement exercé par des traductrices. La même règle est appliquée aux autres métiers. 


\section{AUTEUR}

\section{ÉLISABETH LAVAULT-OLLÉON}

Université Grenoble Alpes, ILCEA4 (GREMUTS) 